\title{
The Role of Structural and Temporal Factors in the Architectural Development of the Mango Tree: Evidences from Simulation
}

\author{
Frédéric Boudon ${ }^{1}$, Alexandra Jestin ${ }^{2}$, Pierre Fernique ${ }^{1}$, Pierre Eric Lauri ${ }^{3}$, Anaëlle \\ Dambreville $^{2}$, Yann Guédon ${ }^{1}$, Frédéric Normand ${ }^{2}$ \\ ${ }^{1}$ CIRAD, UMR AGAP, Inria Virtual Plants team, 34095 Montpellier Cedex 5, France \\ ${ }^{2}$ CIRAD, UPR HortSys, 97455 Saint-Pierre, La Réunion, France \\ ${ }^{3}$ INRA, UMR AGAP, 34095 Montpellier Cedex 5, France
}

Keywords: mango tree, architecture, functional-structural plant model, generalized linear model, vegetative development, flowering.

\begin{abstract}
The mango tree architecture can be defined as an arborescence of vegetative growth units and inflorescences in terminal position. The vegetative and reproductive development of the mango tree is affected by endogenous structural and temporal factors and their interplays. This could explain for example the well-known phenological asynchronisms within and between trees. However, the relative importance of these factors in the architectural development has not yet been assessed. For this purpose, we designed a model of mango tree architectural development. In this model, the appearance of growth units and inflorescences was decomposed into elementary events describing the occurrence, the intensity and the timing of vegetative and reproductive development. The corresponding probabilities were estimated using generalized linear models (GLMs) and were affected by different structural and temporal factors, such as the apical or lateral position, or the vegetative or reproductive fate, of the previous growth unit. GLMs were estimated on the basis of mango tree architectures, cultivar Cogshall, measured during two production cycles in Réunion Island. A model of architectural development was then designed using the L-systems formalism and consisted in successive applications of development rules in order to assemble in parallel new units in different topological positions and with different fates. As a result, a simulation of the model gave an integrative view of the dynamics of the population of growth units and inflorescences of the tree. We could assess in this way more precisely the impact of the structural and temporal factors on the vegetative and reproductive development of the mango tree by comparing simulated with actual tree architectures. Additionally, a 3D representation could be estimated to visualise the generated structures. As a perspective, this model of architectural development is a cornerstone for an integrative crop model of the mango tree including carbon- and water- related ecophysiological processes for mango fruit development (see poster of Gretchi et al. in this symposium).
\end{abstract}

